\title{
katG Gene as a Surrogate Molecular Marker Leading to Cause Drug Resistance in Mycobacterium tuberculosis Isolates
}

\author{
Makeshkumar Veluchamy,*, Radha Madhavan ${ }^{1}$, Sujatha Narayanan², Lakshmi Rajesh ${ }^{2}$ \\ ${ }^{1}$ Department of Microbiology, SRM Medical College Hospital and Research Centre, SRM University, Kancheepuram District, \\ Tamilnadu, India \\ ${ }^{2}$ Department of Immunology, National Institute for Research in Tuberculosis (NIRT) [Formerly Tuberculosis Research Centre], Indian \\ Council of Medical Research (ICMR), Tamilnadu, India \\ *Corresponding author: vmakeshkumar@yahoo.com
}

Received August 20, 2013; Revised August 26, 2013; Accepted September 21, 2013

\begin{abstract}
Multi Drug Resistant Tuberculosis (MDR-TB) is an emerging problem of great importance to public health worldwide. Resistance to Rifampicin (RIF) and Isoniazid (INH) are considered as surrogate markers for MDR-TB. Resistance in Mycobacterium tuberculosis to Rifampicin is reported to be conferred by mutation in an $81 \mathrm{bp}$ rifampicin resistance determining region (RRDR) of the $\beta$ subunit of RNA polymerase encoded by rpoB gene corresponding to codons 507-533. Resistance to Isoniazid is conferred by mutation in Catalase-peroxidase gene (katG), alkyl hydroperoxidase gene $(a p h C)$ and enoyl acyl reductase gene (inhA). The aim of this study was to identify the mutation conferring resistance to INH and RIF by automated DNA sequencing. Seventy-four isolates of M. tuberculosis were tested phenotypically with four anti tuberculosis drugs namely Rifmapicin (RIF), Isoniazid (INH), Ethambutol (EMB) and Streptomycin(SM) by absolute concentration method. Three isolates from pulmonary tuberculosis (PTB) patients were resistant to RIF with minimum inhibitory concentration (MIC) greater than or equal to $128 \mu \mathrm{g} / \mathrm{ml}$ and to INH with MIC greater than are equal to $5 \mu \mathrm{g} / \mathrm{ml}$. The genome of these three MDR-TB isolates were amplified by Polymerase Chain Reaction (PCR) and DNA sequencing was performed. All three isolates showed a point mutation at codon 315 (Ser ${ }^{\circledR}$ Thr) in the sequenced kat $G$ region for INH resistance, but did not show any mutation in the 81bp hotspot sequenced $r p o B$ region for RIF resistance and promoter region of inhA and $a p h C$ for INH resistance. Two of three isolates were from patients who had not taken treatment previously which is of great concern with respect to public health.
\end{abstract}

Keywords: Multi Drug Resistant Tuberculosis, rifampicin/isoniazid, PCR/DNA sequencing

Cite This Article: Makeshkumar Veluchamy, Radha Madhavan, Sujatha Narayanan, and Lakshmi Rajesh, "katG Gene as a Surrogate Molecular Marker Leading to Cause Drug Resistance in Mycobacterium tuberculosis Isolates.” American Journal of Infectious Diseases and Microbiology 1, no. 5 (2013): 86-91. doi: 10.12691/ajidm-1-5-2.

\section{Introduction}

Tuberculosis (TB) remains a public health issue in many parts of the world [1]. The situation is further complicated by the emergence of multidrug-resistant TB (MDR-TB) [2]. MDR-TB is recognized as infection with Mycobacterium tuberculosis resistant to isoniazid and rifampicin. India and China carry almost $50 \%$ of worlds MDR-TB burden [3]. Recent data from the World Health Organization (WHO) estimated that 4, 89,139 MDR-TB cases were prevalent in 2006, arising from a global proportion of $4.8 \%$ of all TB cases (95\% CI 4.6-6.0). Despite the formidable burden, it is estimated that less than $5 \%$ of existing MDR-TB cases are currently being detected [4].

The most effective pharmacotherapy is a multidrug combination of Isoniazid (INH), Rifampicin (RIF), Pyrazinamide (PZA) and Ethambutol (EMB) for an initial two month intensive treatment (initial intensive phase). This is followed by a continuation phase, usually four month treatment with RMP and INH exclusively [5]. INH and RIF, the most potent anti-tuberculosis drugs, kill more than $99 \%$ of tubercle bacilli within two months of initiation of therapy and while any residual dormant bacilli or replicating RIF resistant mutants are eliminated during the continuation phase [6,7]. The complete genome of M.tuberculosis strains has been mapped as a length of about $4.4 \mathrm{Mb}$, however each gene of M.tuberculosis may have separate function including the specific complex formation with important drugs being used as tuberculosis treatment regime. Unusual genetic alteration of drug resistance [8].

The mechanism of action of rifampicin is to inhibit mycobacterial transcription by targeting DNA-dependent RNA polymerase. RNA polymerase is a complex oligomer composed of four different subunits $(\alpha, \beta, \beta$ and $\infty$ encoded by rpo $A$, rpo $B$, rpoC and rpoD) is highly conserved among bacterial species. The development of 
resistance to rifampicin is due to mutations in well-defined, 81 base pair (bp) (27 codons) central region of the gene that encodes the $\beta$-subunit of RNA polymerase (rpoB) [9]. Most reference laboratories that use molecular methods examine only the 81bp region. Previous work indicates that more than $95 \%$ of RMP resistant strains are associated with mutations within an $81 \mathrm{bp}$ region of the $r p o B$ gene. Specific mutations, insertions, and deletions have been detected, and this 81bp region has been termed the rifampicin resistance determinant region [RRDR] [10].

Isoniazid, a first line anti-TB drug, has a simple chemical structure consisting pyridine ring and hyrazide group. INH is a pro drug that enters actively growing tubercle bacilli by passive diffusion [11]. The bifunctional bacterial enzyme Catalase-peroxidase (katG) converts INH to a range of oxygenated and organic toxic radicals that attack multiple targets in the mycobacterial cell wall $[12,13]$. The best characterized target of these radicals is the cell wall mycolic acid, but DNA, carbohydrates, lipids, and DNA metabolism may be targeted as well [14]. Reports suggest that an NADH- dependent by inhA and kas $A$ are involved in the biosynthesis of mycolic acids and are two potential intracellular enzymatic targets for activated INH [15,16]. Mutations in dispersed gene loci including katG (Catalase-peroxidase), the promoter region of aphC (alkyl hydroperoxidase), inhA (enoyl-acyl reductase), kasA (beta ketoacyl ACP synthase), mabA (3ketoacyl reductase), and ndh (NADH dehydrogenase) have been found to associated with INH resistance $[17,18,19,20]$ Mutations in the oxyR-aphC intergenic region, where the putative promoter of $a p h C$ is located, are considered to be a compensatory mechanism for the loss of katG function in resistant strains [19,21]. Sequencing of PCR-amplified products of rpoB and katG has become the most widely used genotypic method for detecting drug resistance in M.tuberculosis; it is accurate and reliable and it has become the reference standard for mutation detection [22]. The aim of this study was to identify the mutation conferring resistance to INH and RIF by automated DNA sequencing method.

\section{Materials and Methods}

\subsection{M.tuberculosis Isolates}

Seventy four clinical isolates of M.tuberculosis were included in this study; sixty-eight isolates from sputum AFB smear positive pulmonary tuberculosis patients samples and six isolates from suspected extra pulmonary tuberculosis patients, clinical specimens included; pus (1), synovial fluid (1) urine (2), fine needle aspirates (2) in SRM Medical College Hospital and Research Centre, Kattankulathur, Kanchipuram District, Tamilnadu, South India during the period of May 2008 to May 2010. Informed written consents were taken from all patients and the study was approved by the Institutional Ethical Committee (IEC), SRM Medical College Hospital \& Research Centre, SRM University, Kattankulathur-603203, Kanchipuram District.

\subsection{Antimycobacterial Susceptibility Assay}

Conventional indirect susceptibility testing was done in Middle brook 7H10 agar medium and tested to four anti-
TB drugs of Rifampicin (RIF), Isoniazid (INH), Ethambutol (EMB) and Streptomycin(SM) and determined by the MIC method. The following critical concentrations of anti-TB drugs were used in this study; $16,32,64,128 \mu \mathrm{g} / \mathrm{ml}$ for RIF, $0.1,0.2,1,5 \mu \mathrm{g} / \mathrm{ml}$ for

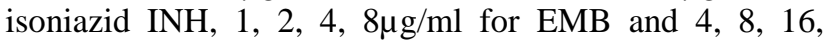
$32 \mu \mathrm{g} / \mathrm{ml}$ for SM. A strain was considered as MDR-TB if the cut off value more than $64 \mu \mathrm{g} / \mathrm{ml}$ for RIF, $1 \mu \mathrm{g} / \mathrm{ml}$ for INH, $4 \mu \mathrm{g} / \mathrm{ml}$ for EMB and $32 \mu \mathrm{g} / \mathrm{ml}$ for SM.

\subsection{DNA Extraction and Polymerase Chain Reaction (PCR)}

Chromosomal DNA was extracted by the conventional CTAB (Cetyl-tri-methyl ammonium bromide) phenol chloroform extraction method [23].

\subsubsection{Primers}

Rifampicin resistance determining region (RRDR) rpoB gene was amplified by using primers rpo3 (5'CAGACGTTGATCAACATCCG 3') and rpo4 (5' TACGGCGTTTCGATGAAC 3') to generate an 803 bp product that contains the 81bp hot spot RRDR. The 209bp katG gene was amplified by using primers such as forward sequence (5'GAAACAGCGGCGCTGGATCGT 3') and reverse sequence (5'GTTGTCCCATTTCGTCGGGG 3'). The 248bp inhA gene was amplified by using primers such as forward sequence (5'CCTCGCTGCCCAGAAAGGGA 3') and reverse sequence (5'ATCCCCCGGTTTCCTCCGGT 3'). The 236bp aphC was amplified by using primers such as forward sequence (5'ACCACTGCTTTGCCGCCACC 3') and reverse sequence (5'CCGATGAGAGCGGTGAGCTG 3’).

\subsubsection{Amplification Master Mix}

Amplification was performed using the mixture containing $1 \mu \mathrm{l}$ of nuclease free water, $1 \mu \mathrm{l}$ of forward and reverse primers $(10 \mathrm{pmol})$ each, $6 \mu \mathrm{l}$ of deoxyribonucleoside triphosphate (d NTP) mix (10Mm), $2.5 \mu \mathrm{l}$ of 10X PCR buffer, 10-50ng of template genomic DNA $(5 \mu \mathrm{l})$ and $1 \mathrm{U}$ of Taq DNA polymerase $(4.5 \mu \mathrm{l})$. Final volume of the sample was $20 \mu \mathrm{l}$.

\subsubsection{Amplification Condition}

The following protocols used for rifampicin and isoniazid resistant genes amplification. The amplification cycles were performed on an automated thermal cycler (Applied biosystem Gene Amp PCR system 9700).

\begin{tabular}{|c|c|c|c|c|}
\hline $\begin{array}{c}\text { Resistant } \\
\text { Genes }\end{array}$ & Denaturation & Annealing & Extension & $\begin{array}{c}\text { No of } \\
\text { cycles }\end{array}$ \\
\hline \multirow{2}{*}{ rpoB } & $94^{\circ} \mathrm{C}$, & $54^{\circ} \mathrm{C}$, & $72^{\circ} \mathrm{C}$ & 40 Cycles \\
\hline \multirow{2}{*}{ katG } & $94^{\circ} \mathrm{C}$, & $63^{\circ} \mathrm{C}$, & $72^{\circ} \mathrm{C}$ & \multirow{2}{*}{30 Cycles } \\
\hline \multirow{2}{*}{ inhA } & $94^{\circ} \mathrm{C}$, & $56^{\circ} \mathrm{C}$, & $72^{\circ} \mathrm{C}$ & \multirow{2}{*}{30 Cycles } \\
\hline \multirow{2}{*}{$\boldsymbol{a p h C}$} & $94^{\circ} \mathrm{C}$, & $55^{\circ} \mathrm{C}$, & $72^{\circ} \mathrm{C}$ & \multirow{2}{*}{30 Cycles } \\
\hline
\end{tabular}

The last cycle was followed by cooling to $4{ }^{\circ} \mathrm{C}$ and holding at this temperature until the tubes were taken out off the machine.

\subsubsection{Detection of Amplified Products}

Amplified products were resolved by agarose gel electrophoresis (2\%) at 80 volts for $40 \mathrm{~min}$. Gel was 
stained with ethidium bromide $(0.5 \mu \mathrm{g} / \mathrm{ml})$ and viewed under UV transilluminator (VILBER-LOURMAT, France, TCP - 20.M).

\subsection{Purification of PCR Products}

Prior to sequencing, the PCR product was purified using PCR purification kits (QIAmp DNA purification mini kit). The amplicons were subjected to electrophoresis. The agrose gel piece containing the amplicons were dissolved in gel extraction (Capture) buffer at $56^{\circ} \mathrm{C}$, vortexed and added to the columns. The tube was briefly centrifuged at $13,000 \mathrm{rpm}$ for 1 minute. $300 \mu$ l of wash buffer was added to the column and centrifuged at 13,000rpm for 1 minute. After discarding the flow through column was spun once to removal residual wash buffer with in the column. The column was then transferred to fresh tube and DNAse free water was added to the column and amplicons were eluted by centrifugation for 1 minute at 13,000rpm. The purification eluant was used for automated sequencing method.

\subsection{DNA Sequencing Method}

Sequencing work was carried out by using an automated DNA sequencer (ABI prism 310 Genetic Analyzer, version 3.1-applied biosystems, USA) using both forward and reverse primers to confirm the results.

\section{Results}

Among seventy-four clinical isolates of M.tuberculosis (M.tb) (sixty-eight isolates of M.tb obtained from sputum AFB smear positive pulmonary tuberculosis (PTB) patients and six isolates of M.tb obtained from extrapulmonary tuberculosis patients) tested for drug susceptibility against first-line anti-TB drugs (RIF, INH, EMB, SM), seventy-one (95.9\%) isolates were found to be sensitive to all the four anti-TB drugs. Three $(4.1 \%)$ isolates showed resistance to two anti-TB drugs (RIF, INH). For therapy guidance, isolates with 64 or $128 \mu \mathrm{g} / \mathrm{ml}$ for RIF and 1 or $5 \mu \mathrm{g} / \mathrm{ml}$ for INH reported as intermediate resistant. For national and international surveillance of antituberculosis drug resistance, intermediate-resistant isolates were reported as resistant. So totally, three phenotypically resistant isolates were selected on the basis of minimum inhibitory concentration (MIC) value greater than or equal to $128 \mu \mathrm{g} / \mathrm{ml}$ for RIF resistance and INH with MIC greater than or equal to $5 \mu \mathrm{g} / \mathrm{ml}$ from AFB smear positive PTB patients. Three MDR-TB pulmonary isolates were successfully amplified the following RIF and INH drug resistant genes were; $\beta$-subunit of RNA polymerase encoded $r p o B$ gene, Catalase-peroxidase encoded katG gene, alkyl hydroperoxidase encoded aphC gene and enoyl acyl reductase encoded inhA gene by PCR assay (Figure 1, Figure 2 and Figure 3).

All three MDR-TB isolates showed a point mutation at codon 315 (Ser@ Thr) in the sequenced katG region for INH resistance (Figure 4). In addition, a strong correlation was found between the $\mathrm{MIC}_{\mathrm{S}}$ of $\mathrm{INH}$ and the level of mutation in the isolates. The high MIC values of $\geq 5 \mu \mathrm{g} / \mathrm{ml}$ of three isolates had AGC-ACC nucleotide alteration but no mutation was detected in the promoter region of inhA and $a p h C$ by sequencing method for INH resistance. No mutation was detected in the 81bp hot-spot RRDR in three MDR-TB isolates by sequencing method and this result correlated with high MIC value of $128 \mu \mathrm{g} / \mathrm{ml}$ for RIF resistance. However, not all mutations within the 81bp hot-spot region exhibit the same level of resistance. Most of the reference laboratories that use molecular methods examine only the 81 hot-spot bp region.

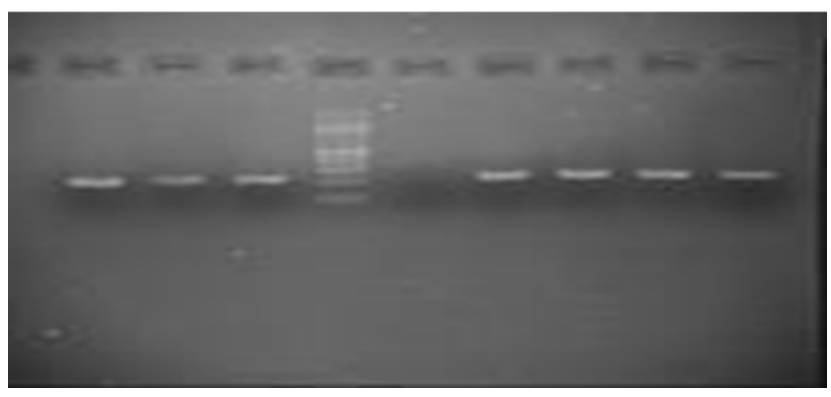

Figure 1.

(From right side lane 1, 2 and 3 - 236bp aphC gene amplified in three MDR-TB isolates, lane 4 - Positive control of Mycobacterium tuberculosis $\mathrm{H}_{37} \mathrm{R}_{\mathrm{V}}$, lane 5 Negative Control, Lane 6 - 1Kb Molecular Marker, lane 7,8 and 9 - 209bp katG gene amplified in three MDR-TB isolates).

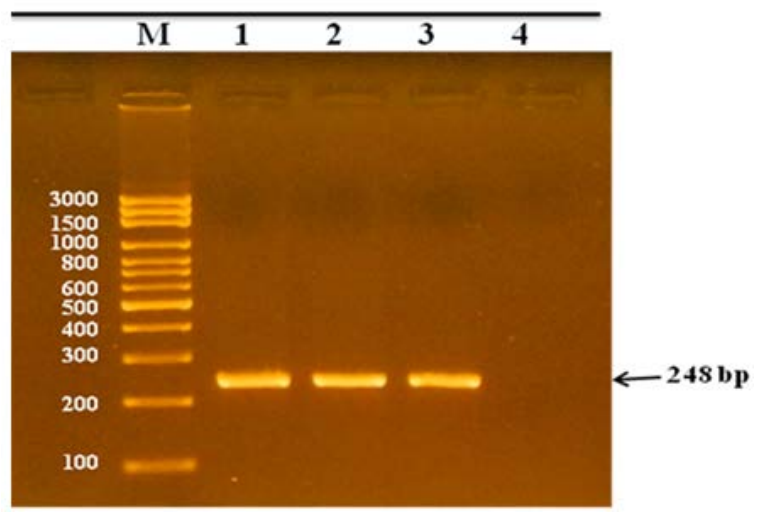

Figure 2.

(M - 1Kb Molecular Marker, Lane 1, 2 and 3 - 248bp inhA gene amplified in three MDR-TB isolates)

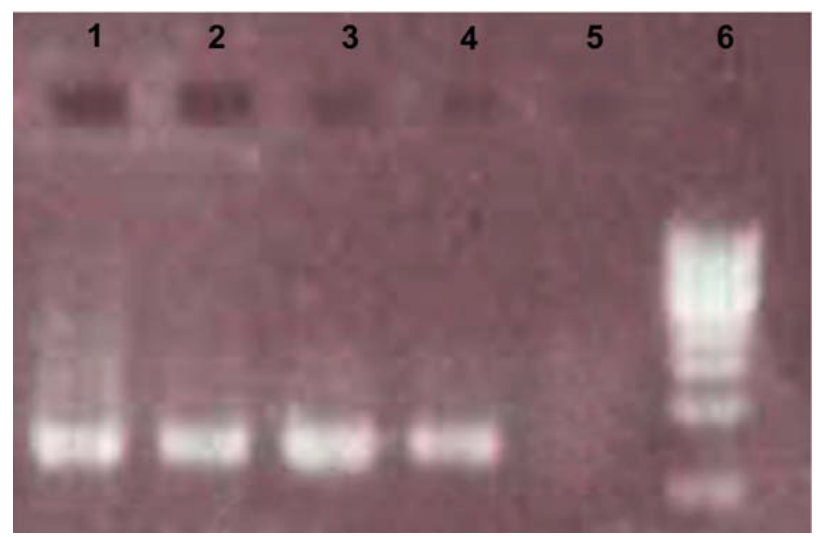

Figure 3.

Lane 1, 2 and $3-803$ bp hotspot RRD region of rpoB gene amplified in three MDR-TB isolates, Lane 4 Positive Control of Mycobacterium tuberculosis $\mathrm{H}_{37} \mathrm{R}_{\mathrm{V}}$, lane 5 - Negative Control, Lane 6 - 1Kb Molecular Marker. 

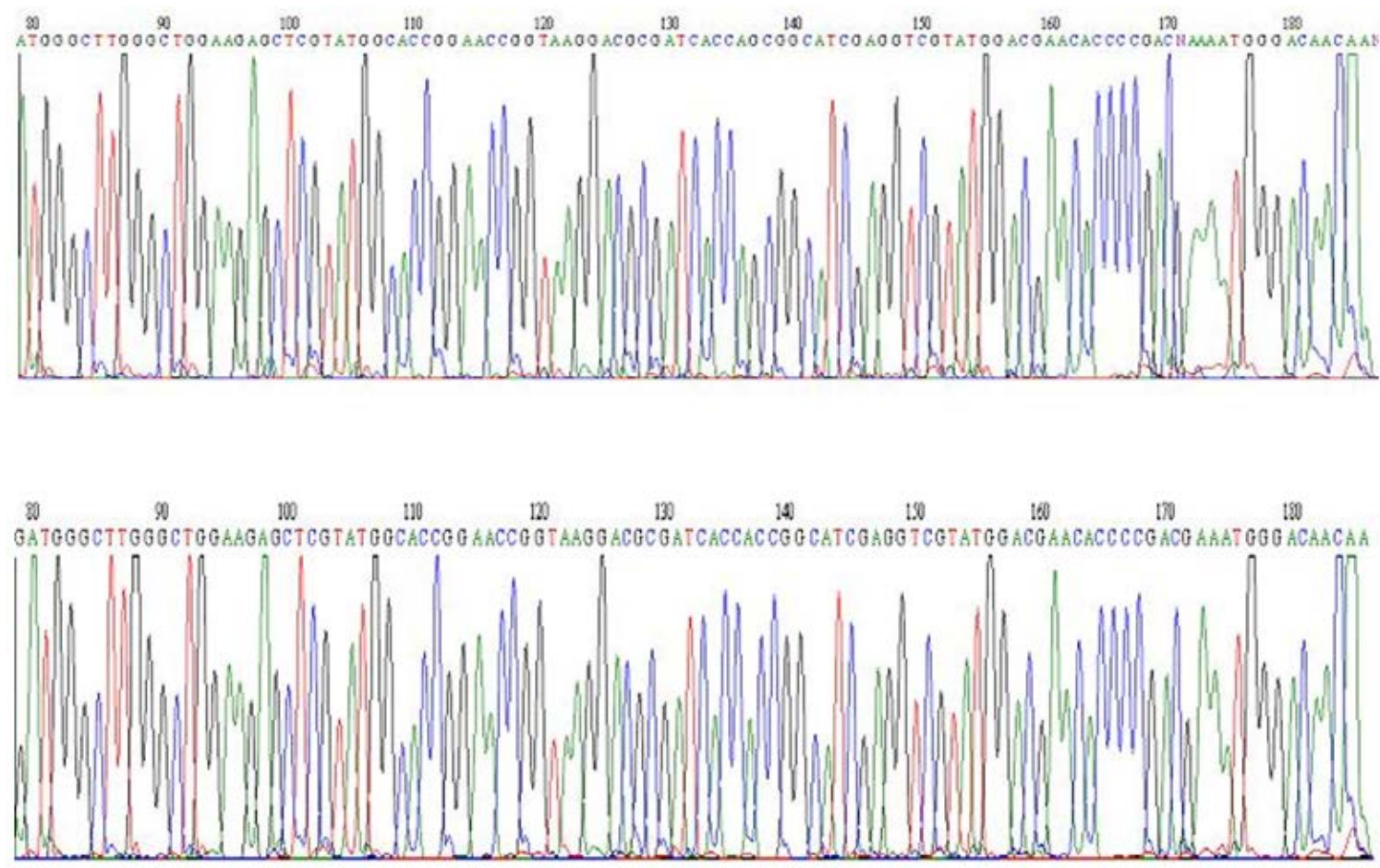

Figure 4.

\section{Discussion}

The three to five percentage of RIF resistant isolates do not possess any mutations in the $r p o B$ gene [24]. The mutation responsible for RIF resistance in these strains could be located either outside the region of analysis or changes that might have occurred in genes whose product participate in antibiotic permeation or metabolism [25]. There are also reports from India on mutations outside the 81bp hotspot region [26]. Mercy Aparna et al., found one out of 34 M.tuberculosis isolates from Koraput; showed rifampicin resistance and this isolate had no mutation in RRDR and outside of hotspot region [27]. Report of a RIF resistant strain from Australia indicated a mutation outside the core region of $r p o B$ [28]. In other similar studies, Khamis et al., found ten out of the eleven rifampicin resistant specimens (90.9\%) showing gene mutation versus only one $(9.1 \%)$ with no mutation. The molecular mechanism of resistance in $4 \%$ of RIF resistant tuberculosis isolates that lack RRDR changes is unknown [29]. An another explanation was hetero resistance; that means the presence of mixture of susceptible and resistant subpopulation in culture which could be an obstacle against the sensitivity of molecular drug resistance testing and successive therapy [30].

The INH resistance mechanism is more complex and not as well understood as the mechanism responsible for RIF resistance. Currently 80 to $90 \%$ of INH resistance phenotypes have been discovered to be associated with mutations in KatG, inhA, aphC and ndh genes. INH resistance occurs due to substitutions in the Catalaseperoxidase kat $G$ gene in more than three quarters of cases and more rarely, due to mutation in inhA (less than 10\%) and the aphC gene. The predominant mode of acquisition of resistance via katG alteration is the selection of particular mutation i.e. the transversion of codon 315 AGC-ACC (Ser-Thr) that decrease the catalase activity, but maintains a certain level of the peroxidase activity of the enzyme in viable INH-resistant organisms [31].

In 60 to $70 \%$ of the cases, INH resistance is associated with mutations in the Catalase-peroxidase coding katG gene at codon 315(Ser-Thr) [32]. The majority of hot mutation in katG gene of M.tuberculosis have been reported in codon 315(Ser-Thr) and less in other codons [33]. Mutation at codon 315 of KatG gene is most prevalent in isoniazid resistant Mycobacterium tuberculosis and is high in area with high TB incidence. The prevalence of mutations at codon 315 varies greatly in different geographical regions [34].

Several studies have revealed that mutation in KatG gene is responsible for $60-70 \%$ of INH resistant strains [35]. A study by Negi et al., in India revealed KatG S315T mutation in $74.19 \%$ of strains of MTB from Delhi [36]. Similar study conducted by Bhatt CP et al., in Nepal revealed point mutation in 9 (45\%) new and 8 (40\%) old PTB cases from 20 MDR-TB strains [8].

In contrast the Ser315Thr mutation accounted for INH resistance in 52-64\% of strains in Central Africa [31,37]. Similarly, Wang Y.C et al., from China reported $68.6 \%$ [38]; Hass W.H [37] from West Africa reported 60\% of INH resistant strains associated with mutation in KatG due to S315T substitution is similar to those reported in other parts of the world, which reflects a global pattern. Furthermore, in current study association was conformed between the common KatG Ser315Thr mutation in INH resistant strains and the presence of resistance to other first line drugs (RIF, EMB, SM). Hu Yi et al., in their study correlation similar between INH resistance and development of resistance to other first line drugs [39].

The molecular mechanisms responsible for the incidence of MDR-TB in Kattankulathur Village, Kanchipuram district have been reported first time in this hospital area. Although current MDR-TB rates are low in this area and major limitation to molecular genetic detection of drug resistance by any technique is that 
molecular genetic tests detect only known mutation. Prevalence of mutations may vary by geographical area, identification of a resistance associated mutation can be informative, but lack of a mutation in the target sequence must be interpreted with caution.

\section{Conclusion}

Majority of the MDR resistant isolates of M.tuberculosis showed common point mutations in the katG gene. Our study also revealed mutation in katG gene was Ser315Thr (S315T) and thus serves as potential genetic marker for the detection of INH resistance. The absence of mutation in $81 \mathrm{bp}$ hotspot $r p o B$ region of cluster II in three isolates may be attributed to the presence of mutation in a region outside the specific sequence of the primers in the $3.5 \mathrm{~kb} r p o B$ region. In this study, an additional interesting finding was that three isolates were showed MIC of $128 \mu \mathrm{g} / \mathrm{ml}$ for rifampicin drug. This resistant was determined by the conventional drug sensitivity (absolute concentration) method. But sequencing analysis did not find any mutation within the 81bp hot spot core region. The genomic mutation responsible for RIF resistance in this study must be lying outside of the 81bp hotspot RRDR region. The possibilities are that, in this resistant isolates could be attributed other mechanism of resistance may be involved. Previous studies reported 3-5\% of rifampicin isolates that lack of RRDR region. Comparative to previous report, this study also supported to gives the additional information of unknown rifampicin resistance mechanism.

\section{Acknowledgement}

Financial assistance in the form of a university research fellowship (URF) provided by the SRM Medical College Hospital and Research Centre, SRM University, Kattankulathur-603203, Kancheepuram District, Tamilnadu, South India.

\section{Conflict of interest}

None declared.

\section{Source of Support}

University Research Fellowship.

\section{References}

[1] World Health Organization.(2005).Global tuberculosis controlsurveillance, planning, financing. World Health Organization, Geneva, Switzerland. WHO/HTM/TB/2005.349.

[2] Toungoussova O., Sandven P., Mariandyshev A., et al. (2002).Spread of drug-resistant Mycobacterium tuberculosis strains of the Beijing genotype in the Archangel Oblast, Russia. J Clin Microbiol 40, 1930-1937.

[3] Wright A, Zingol M, Deun A.V et al. (2009). Epidemiology of anti-tuberculosis drug resistance 2002-07: an updated analysis of the global project on anti-tuberculosis drug resistance surveillance. The Lancet, 373, 1861-72.
[4] World Health Organization. Anti-tuberculosis Drug Resistance in the World. Report No.4. Pre-publication version. Available from: whqlibdoc.who.int/hq/2008/WHO_HTM_TB_2008.394_eng.pdf, Accessed 5 May 2008.

[5] Onyebujoh P, Zumla A, Ribeiro I, et al. (2005). Treatment of tuberculosis: Present status and future prospects. Bulletin of World Health Organization 83, 857-865.

[6] Mitchison DA. (1985). Mechanism of drug action in short-course chemotherapy. Bulletin International Union against Tuberculosis 65, 30-37.

[7] Iseman MD, Madsen LA. (1989). Drug resistant tuberculosis. Clin Chest Med., 10, 341-5.

[8] Bhatt CP, Bhatt AB, Shrestha B. (2011). Molecular characterization and comparison of multidrug resistant strains of Mycobacterium tuberculosis by phenotypic and genotypic method. SARRC J Tuber Lung Dis HIV/AIDS, VIII(2).

[9] Telenti A., Imboden P., Marchesi F., et al. (1993). Detection of rifampicin-resistance mutations in Mycobacterium tuberculosis. Lancet ., 341, 647-650.

[10] Yue J., Shi W., Xie J., et al. (2003). Mutation in the rpoB gene of multidrug-resistant Mycobacterium tuberculosis isolates from China. J Clin Microbiol., 41, 2209-2212.

[11] Bardou FC., Raynaud C., Ramos MA., et al. (1998). Mechanism of isoniazid uptake in Mycobacterium tuberculosis. Microbiology144:2539-2544.

[12] Rozwarski DA., Grant GA., Barton DHR., et al. (1998). Modification of the NADH of the isoniazid target (inhA) from Mycobacterium tuberculosis. Science., 279, 98-102.

[13] Sacchettini JC, and Blanchard JS. (1996). The structure and function of the isoniazid target in Mycobacterium tuberculosis. Res Microbiol., 147, 36-43.

[14] Cardoso RF., Cooksey RC., Morlock GP., et al. (2004). Screening and characterization of mutations in isoniazid-resistant Mycobacterium tuberculosis isolates obtained in Brazil. Antimicrob. Agents Chemother., 48, 3373-3381.

[15] Marrakchi H, Laneelle G and Quemard A. (2000). InhA, a target of the antituberculosis drug isoniazid is involved in a mycobacterial fatty acid elongation system, Fas-II. Microbiology., 146, 289-296.

[16] Mdluli K., Slayden RA., Zhu Y., et al. (1998). Inhibition of a Mycobacterium tuberculosis_-ketoacyl ACP synthase by isoniazid. Science., 280, 1607-1610.

[17] Banerjee A., Dubnau E., Quemard A., et al. (1994). inhA, a gene encoding a target for isoniazid and ethionamide in Mycobacterium tuberculosis. Science., 263, 227-230.

[18] Ducasse-Cabanot S., Cohen-Gonsaud M., Marrakchi H., et al. (2004). In vitro inhibition of the Mycobacterium tuberculosis $\beta$ ketoacyl-acyl carrier protein reductase MabA by isoniazid. Antimicrob Agents Chemother., 48, 242-249.

[19] Kelley CL., DA Rouse., and Morris S L., (1997). Analysis of $a h p C$ gene mutations in isoniazid-resistant clinical isolates of Mycobacterium tuberculosis. Antimicrob Agents Chemother., 41, 2057-2058.

[20] Miesel L., Weisbrod TR., Marcinkeviciene JA., et al. (1998). $\mathrm{NADH}$ dehydrogenase defects confer isoniazid resistance and conditional lethality in Mycobacterium smegmatis. J Bacteriol., 180, 2459-2467.

[21] Wilson T., Lisle GW., Marcinkeviciene JA., et al. (1998). Antisense RNA to $a h p C$, an oxidative stress defense gene involved in isoniazid resistance, indicates that $a$ hpC of Mycobacterium bovis has virulence properties. Microbiology., 144, 2687-2695.

[22] Victor TC., Van Helden PD., (2001). Detection of mutations in Mycobacterium tuberculosis by a Dot Blot Hybridization Strategy. In: Mycobacterium tuberculosis Protocols. Methods in Molecular Medicine., New Jersey: Humana press Vol.54, 155-64.

[23] Somerville W., Thibert L., Schwartzman K., et al. (2005). Extraction of Mycobacterium tuberculosis DNA: A question of containment. J Clin Microbiol., 43, 2996-7.

[24] Ramasamy S., Musser JM., (1998).Molecular genetic basis of antimicrobial agent resistance in Mycobacterium tuberculosis: 1998 update. Tubercle and Lung diseases., 79, 3-29.

[25] Telenti A., Imboden P., Marchesi F., et al. (1993). Direct automated detection of rifampin resistant $M$. tuberculosis by polymerase chain reaction and single strand conformation polymorphism analysis. Antimicrob Agents Chemother., 37, 20548. 
[26] Mani C., Selvakumar N., Sujatha N., et al. (2001). Mutations in the rроB Gene of multi-drug resistant Mycobacterium tuberculosis Clinical isolates from India. J. Clin.Microbiol., 39, 2987-2990.

[27] Mercy Aparna L., Lingala., Aparna Srikantam., et al. (2010). Clinical and geographical profiles of rpoB gene mutations in Mycobacterium tuberculosis isolates from Hyderabad and Koraput in India. Journal of Microbiology and Antimicrobials., Vol.2(2),pp.13-18.

[28] Yuen Lily KW., Leslie David., Colol Peter J., (1999). Bacteriological and molecular analysis of rifampicin resistant Mycobacterium tuberculosis strains isolated in Australia. J Clin Microbiol., 37, 3844-3850.

[29] Khamis N., Amin MM., Zagloul MZ., et al. (2001). DNA sequencing and bacteriophage based technique for rapid detection of rifampicin resistant Mycobacterium tuberculosis. Egypt J Med Lab Sci (ESIC)., 13(1), 28-36.

[30] Heep M., Brandstatter B., Riegger U., et al. (2001), Frequency of $r p o B$ mutation inside and outside the cluster I region in rifampinresistant clinical M.tuberculosis isolates. J Clin Microbiol., 39, 107-10.

[31] Dobner P., Rusch - Gerdes S., Bretzel G., et al. (1997). Usefulness of M.tuberculosis genomic mutation in the genes KatG and inhA for the prediction of isoniazid resistant. Int J Tubercl Lung Dis., 1, 365.

[32] Heym B., Zhang Y., Poulet S., et al. (1993). Characterization of the katG gene encoding a Catalase-peroxidase required for isoniazid susceptibility of Mycobacterium tuberculosis. J. Bacteriol., 13, 4255-4259.
[33] Zheltokjva EA., Chemousova LN., Smiirnova TJ., (2004). Molecular genetic typing Mycobacterium tuberculosis strains isolated from patents in the samapa region by the restriction DNA fragment length polymorphisms ZH. Microbial (Moscow), 5, 3943.

[34] Siqueria de R.H., Freitas de D.A.F., Oliveria de N.D., et al. (2009) Isoniazid resistant Mycobacterium tuberculosis strains arising from mutations in two different regions of the KatG gene. J. bras.pneumol., 35(8), 773-9.

[35] Aragon L.M., Navarro F., Heiser V., et al. (2006). Rapid detection of specific gene mutations associated with isoniazid or rifampicin resistance in Mycobacterium tuberculosis clinical isolates using non-fluroscent low density DNA microarrays. Journal of Antimicrobial Chemotherapy, 57, 825-83.

[36] Negi S.S., Anand R., Pasha S.T., et al. (2006). Molecular characterization of mutation associated with rifampicin and isoniazid resistance in Mycobacterium tuberculosis isolates. Indian Journal of Experimental Biology., 44, 547-53.

[37] Hass W.H., Schilke K., Brand J., et al. (1997). Molecular analysis of KatG gene mutations in strains of Mycobacterium tuberculosis complex from Africa. Antimicro Agents Chemother., 1601-3.

[38] Wang Y.C, Zhu R.Y, Xu Y.Y, et al. (2009). Molecular characterization of drug resistant Mycobacterium tuberculosis isolates in Guangdong, China. Jpn.J.Infect dis, 62, 270-4.

[39] Hu Y., Hoffner S., Weili J., et al. (2010). Extensive transmission of isoniazid resistant M.tuberculosis and its association with increased multidrug resistant TB in two rural countries of eastern China: A molecular epidemiological study. BMC Infectious Diseases, 10(43). 